\title{
Neuropsychiatric features of corticobasal degeneration
}

\author{
Irene Litvan, Jeffrey L Cummings, Michael Mega
}

Neuropharmacology Unit, Defense and Veteran Head Injury Program, Jackson Foundation and the Medical Neurology Branch

I Litvan

National Institutes of Neurological Disorders and Stroke, National Institutes of Health, Bethesda, Maryland, USA

I Litvan

Department of Neurology

J L Cummings

M Mega

Department of Psychiatry and Biobehavioral Science, UCLA School of Medicine, Los Angeles, CA, USA

J L Cummings

Correspondence to: Dr Irene Litvan, NINDS, NIH, Federal Building, Room 714, Bethesda, MD 20892-9130, USA

Telephone 001301496 1189; fax 0013014962358

Received 2 February 1998 and in revised form 27 April 1998

Accepted 7 May 1998

\begin{abstract}
Objective-To characterise the neuropsychiatric symptoms of patients with corticobasal degeneration (CBD).

Methods-The neuropsychiatric inventory (NPI), a tool with established validity and reliability, was administered to 15 patients with CBD (mean (SEM), age 67.9 (2) years); 34 patients with progressive supranuclear palsy (PSP) (66.6 (1.2) years); and 25 controls (70 (0.8) years), matched for age and education. Both patient groups had similar duration of symptoms and mini mental state examination scores. Semantic fluency and motor impairment were also assessed. Results-Patients with CBD exhibited depression $(73 \%)$, apathy $(40 \%)$, irritability $(20 \%)$, and agitation $(20 \%)$ but less often had anxiety, disinhibition, delusions, or aberrant motor behaviour (for example, pacing). The depression and irritability of patients with CBD were more frequent and severe than those of patients with PSP. Conversely, patients with PSP exhibited significantly more apathy than patients with CBD. The presence of high depression and irritability and low apathy scale scores correctly differentiated the patients with CBD $88 \%$ of the time. The irritability of patients with CBD was significantly associated with disinhibition $(r=0.85)$ and apathy $(r=0.72)$. In CBD, apathy was associated with disinhibition $(r=0.67)$; disinhibition was associated with aberrant motor behaviour $(r=0.68)$ and apathy $(r=0.67)$; and aberrant motor behaviour with delusions $(r=1.0)$. On the other hand, depression was not associated with any other behaviour, suggesting that it has a different pathophysiological mechanism. Symptom duration was associated with total motor scores $(r=0.69)$. However, total motor score was not associated with any behaviour or cognitive scores.
\end{abstract}

Conclusions-The findings indicate that frontosubcortical pathways mediating cognition, emotion, and motor function in CBD are not affected in parallel. Patients with CBD and PSP have overlapping neuropsychiatric manifestations, but they express distinctive symptom profiles. Evaluating the behavioural abnormalities of parkinsonian patients may help clarify the role of the basal ganglia in behaviour. (F Neurol Neurosurg Psychiatry 1998;65:717-721)

Keywords: corticobasal degeneration; progressive supranuclear palsy; neurobehaviour
Corticobasal degeneration (CBD) is a neurodegenerative disorder characterised clinically by steadily progressive motor (for example, dystonia, asymmetric parkinsonism not benefitting from levodopa therapy, myoclonus, balance disturbances, and pyramidal signs) and cognitive (for example, ideomotor apraxia, alien hand syndrome, aphasia, visual and sensory neglect) disturbances presenting after the age of $40 .{ }^{12}$ Neuropathologically, it features circumscribed parietal or frontoparietal atrophy, severe cortical neuronal loss, and intense astrogliosis, spongiosis, swollen and achromatic neurons (ballooned cells), neuropil threads, and occasionally, neurofibrillary tangles. In addition, basophilic argyrophilic and tau protein positive inclusions are found in the neurons of the substantia nigra and basal ganglia (subthalamic nucleus, striatum, and pallidum), and also may be present along the dentatorubrothalamic tracts. Feany and Dickson ${ }^{3}$ recently described the "astrocytic plaque" as being pathognomonic of this disorder. Clinical and neuropathological variations have been described which overlap with progressive supranuclear palsy (PSP) and Pick's disease. ${ }^{124-9}$ Several studies have characterised the motor and cognitive features of CBD,${ }^{10-14}$ but the patients' neuropsychiatric symptoms have rarely been described. ${ }^{15} 16$

Neuropsychiatric symptoms are important predictors of burden of the caregiver and the potential for admitting patients to institutions. ${ }^{17-20}$ Thus identification and treatment of the associated behavioural abnormalities may be relevant for improving the quality of life of patients with CBD and their caregivers. In addition, increasing insight into the neurobiological basis of neuropsychiatric disorders facilitates understanding how behaviourally relevant circuits are involved in CBD.

We administered the neuropsychiatric inventory (NPI), a tool with established validity and reliability, to assess the behavioural abnormalities of patients with CBD. ${ }^{21}$ The NPI evaluates both the frequency and severity of 10 behaviours that are commonly reported in patients with cognitive disturbances: delusions, hallucinations, agitation, depression, anxiety, euphoria, apathy, disinhibition, irritability, and aberrant motor behaviour (for example, pacing). To better characterise the behavioural aspects of patients with CBD and to search for distinctive behavioural characteristics, we compared their performance on the NPI to that of a group of patients with PSP of similar age, education, and cognitive impairment and to age matched normal controls. 
Table 1 Demographics of study group

\begin{tabular}{llll}
\hline Characteristics & $\begin{array}{l}\text { Corticobasal } \\
\text { degeneration } \\
(n=15)\end{array}$ & $\begin{array}{l}\text { Progressive } \\
\text { supranuclear } \\
\text { palsy }(n=34)\end{array}$ & $\begin{array}{l}\text { Healthy } \\
\text { controls } \\
(n=25)\end{array}$ \\
\hline Age (y) & $67.9(2)$ & $66.6(1.2)$ & $70(0.8)$ \\
Sex & $8 \mathrm{M} / 7 \mathrm{~F}$ & $23 \mathrm{M} / 11 \mathrm{~F}$ & $14 \mathrm{M} / 11 \mathrm{~F}$ \\
Education (y) & $14.3(0.8)$ & $14.4(0.5)$ & $13.2(0.4)$ \\
Duration (months) & $46.5(5.7)$ & $52(4.5)$ & - \\
Mini mental state examination score & $26.1(1.2)$ & $26(0.8)^{\star}$ & $28.6(0.3)$ \\
Total Mattis dementia rating scale score† & $120.5(4.2)$ & $118.6(2.4)$ & - \\
Total UPDRS motor scores & $26.2(2)^{\star}$ & $21.2(1.3)$ & - \\
\hline
\end{tabular}

Data are presented as mean (SEM). UPDRS=unified Parkinson's disease rating scale score; UPDRS maximum score was 54, and included only motor evaluation of axial and most affected limb (bradykinesia, rigidity, tremor, or dystonia).

* Patients with progressive supranuclear palsy had a significantly lower (worse) mini mental state examination score than controls (ANOVA, $\mathrm{p}<0.05$ ), patients with corticobasal degeneration had a higher (worse) total motor score than those with progressive supranuclear palsy $(p<0.05)$. The score of normal controls, not tested in this study with the Mattis dementia rating scale score, is $\geqslant 136$. $†$ This analysis included 12 patients with corticobasal degeneration and 28 with progressive supranuclear palsy.

\section{Subjects and methods}

Fifteen patients with CBD and 34 with PSP, presenting to the National Institutes of Neurological Disorders and Stroke (NINDS) as outpatients, participated in the study (table 1). The patients with CBD were diagnosed according to the modified criteria of Lang et al. ${ }^{4}$ which included a progressive course of an asymmetric parkinsonism not benefiting from levodopa therapy; the presence of either a dystonic limb or focal myoclonus; the presence of either ideomotor apraxia, alien limb syndrome, or cortical sensory loss; and absence of resting tremor, autonomic disturbance, or laboratory evidence of other disorders. The diagnosis of one of the 15 patients with CBD was later confirmed by necropsy. The PSP study subjects met the NINDS-Society for PSP, Inc (NINDSPSP) clinical research criteria and 22 have previously been reported on. ${ }^{22}$

Twenty five normal controls of the same age and educational levels were recruited at the University of California, Los Angeles (table 1). ${ }^{21}$ Exclusion criteria for all subjects and patients comprised history of alcohol or substance misuse, head trauma with loss of consciousness, and psychiatric disorder preceding the onset of current disease. All participating subjects gave institutional consent. Caregivers and participating subjects were interviewed with the NPI, but as previously described, ${ }^{21}$ the NPI is based on caregivers' information. Briefly, screening questions for each behaviour were posed first, and if a positive response was obtained for one of the 10 neuropsychiatric domains, then this aspect was further explored with scripted questions. The behaviours were rated using a scale for the frequency ( 1 being occasionally, 2 often, 3 frequently, and 4 very frequently) and for the severity ( 1 being mild, 2 moderate, and 3 marked). The composite score for each behavioural domain is the product of the frequency and severity subscore for that particular behaviour (maximum 12). The total score of the NPI is the sum of the subscales scores. In addition, we administered the mini mental state examination (MSSE) $)^{23}$ to all subjects, and the Mattis dementia rating scale $^{24}$ to 12 of the 15 patients with CBD and to 28 of 34 with PSP. There were no significant differences between patients that were administered the Mattis dementia rating scale and those that were not, (age, education, MMSE scores, symptom duration, and motor scores). Verbal fluency (semantic fluency: number of names of items from a supermarket and phonemic fluency: number of words starting with the letter " $F$ ", produced in one minute), and motor impairment (modified unified Parkinson's disease rating scale total motor score (UPDRS) ${ }^{25}$ ) were assessed in the patients. We computed the motor function axially and in the most affected limb. Both patient groups had similar symptom duration, MMSE, and Mattis dementia rating scale scores, but the patients with CBD had a higher UPDRS total motor score (table 1).

Statistical analyses included bootstrap analysis, analysis of variance (ANOVA), logistic regression, and discriminant function analyses. A histogram of NPI data disclosed that composite scores do not generate a normal distribution. Multiplying the frequency (1-4) by the severity (1-3) subscores will not produce a 5,7 , or 11 composite score. The use of nonparametric analysis (for example, the MannWhitney $U$ test) partially accommodates such skewed data but results in a loss of power. Because NPI data generate a non-normal distribution, precluding traditional parametric analysis, a bootstrap analysis ${ }^{26}$ was employed. The program Resampling Stats ${ }^{\circledR}$ was used to evaluate significant differences among patient groups' mean composite scores for each of the 10 NPI behaviours. Bootstrap analysis combines the raw composite scores for any given behaviour of the entire dataset and randomly samples a number of these scores equal to the number making up the groups comprising the data set. A mean difference composite score is then calculated from the randomly comprised samples. This is then repeated 1000 times on the dataset, producing a distribution of possible mean difference composite scores. The mean differences found can then be compared to this distribution of the possible mean difference composite scores between two clinical groups for each behaviour on the NPI. The probability of finding the observed mean difference based on the mean difference generated by resampling is then recorded. This process was repeated 10 times for each of the 10 NPI behaviours to arrive at an average probability value for each comparison. If the observed difference was greater than $95 \%$ of the differences expected from random resampling in the bootstrap method, it was judged to be significant at the 0.05 level.

To explore the relations among the different behaviours, symptom duration, and motor and overall cognitive functions, Spearman correlation coefficients were calculated for the composite scores of these, in a $14 \times 14$ matrix for all patients with CBD (table 2). This exploratory analysis was not meant to establish strict significance, and thus a type 1 error was deemed preferable to missing a possible correlation (type 2 error). For this reason, no Bonferroni adjustment was made; however, an adjusted $\alpha$ level of 0.01 was required for significance. 
Table 2 Correlations of neuropsychiatric inventory (NPI) composite scores, motor, and global cognitive scores in corticobasal degeneration

\begin{tabular}{|c|c|c|c|c|c|c|c|c|c|c|c|c|c|}
\hline & Depression & Apathy & Irritability & Agitation & Anxiety & Disinhibition & Delusions & $\begin{array}{l}\text { Aberrant } \\
\text { motor } \\
\text { behaviour }\end{array}$ & $\begin{array}{l}\text { Total } \\
\text { NPI }\end{array}$ & Duration & $\begin{array}{l}\text { Motor } \\
\text { score }\end{array}$ & $M M S E$ & $M D R S$ \\
\hline \multicolumn{14}{|l|}{ Depression } \\
\hline Apathy & -0.05 & & & & & & & & & & & & \\
\hline Irritability & 0.03 & $0.72^{\star \star}$ & & & & & & & & & & & \\
\hline Agitation & 0.05 & 0.10 & 0.25 & & & & & & & & & & \\
\hline Anxiety & 0.26 & $0.62 \dagger$ & 0.33 & 0.36 & & & & & & & & & \\
\hline Disinhibition & -0.23 & $0.67^{\star}$ & $0.85^{\star \star \star}$ & 0.36 & 0.42 & & & & & & & & \\
\hline Delusions & 0.03 & 0.49 & $0.58+$ & $0.62 \dagger$ & $0.68^{\star \star}$ & $0.68^{\star \star}$ & & & & & & & \\
\hline \multicolumn{14}{|l|}{ Aberrant motor } \\
\hline behaviour & 0.03 & 0.49 & $0.58 \dagger$ & $0.62 \dagger$ & $0.68^{\star \star}$ & $0.68^{\star \star}$ & $1.0^{\star \star \star}$ & & & & & & \\
\hline Total NPI score & $0.60 \dagger$ & $0.58 \dagger$ & $0.67^{\star}$ & 0.31 & 0.55 & $0.59 \dagger$ & 0.44 & 0.44 & & & & & \\
\hline Duration & 0.15 & 0.13 & 0.31 & 0.22 & 0.27 & $0.53 t$ & 0.31 & 0.31 & 0.51 & & & & \\
\hline Motor score & 0.26 & -0.03 & 0.18 & 0.17 & 0.36 & $0.27^{\circ}$ & 0.31 & 0.31 & 0.35 & $0.69^{\star \star}$ & & & \\
\hline MMSE & 0.01 & 0.01 & 0.37 & 0.25 & -0.02 & 0.48 & 0.41 & 0.41 & 0.29 & 0.51 & 0.50 & & \\
\hline MDRS & -0.26 & -0.22 & 0.31 & -0.13 & -0.30 & 0.31 & - & - & -0.12 & 0.27 & 0.39 & $0.78^{\star \star}$ & \\
\hline Verbal fluency & -0.23 & $-0.78 \dagger$ & -0.65 & -0.12 & -0.58 & -0.42 & -0.49 & -0.49 & -0.64 & 0.12 & 0.13 & 0.07 & 0.55 \\
\hline
\end{tabular}

Adjusted $\alpha=0.01$ was required for significance. $\nmid \mathrm{p}<0.03 ;{ }^{\star} \mathrm{p} \leqslant 0.01 ;{ }^{\star \star} \mathrm{p} \leqslant 0.005 ;{ }^{\star \star \star} \mathrm{p} \leqslant 0.001$

\section{Results}

Patients with CBD exhibited depression $(73 \%)$, apathy $(40 \%)$, irritability $(20 \%)$ and agitation $(20 \%)$, but rarely $(\leqslant 14 \%)$ anxiety, disinhibition, delusions, or aberrant motor behaviour (table 3). No patient with CBD manifested euphoria or hallucinations.

The depression and irritability of patients with CBD were significantly more frequent and severe than those of patients with PSP and normal controls. However, those with PSP exhibited significantly more apathy. The other behaviours were similar in the two patient groups (table 3 ).

Logistic regression analysis indicated a significantly higher frequency of depression $\left(\chi^{2}=13, p<0.001\right.$, odds ratio $\left.=1299\right)$ and irritability $\left(\chi^{2}=15.4, \mathrm{p}<0.001\right.$; odds ratio $\left.=27065\right)$ and lower apathy $\left(\chi^{2}=22 ; \mathrm{p}<0.0001\right.$; odds ratio $=0.0001)$ in patients with CBD compared with patients with PSP (overall model, 39.4; $\mathrm{p}<0.0001)$. A discriminant function analysis performed on the patient data set (CBD and PSP) disclosed that any patient with high depression and irritability scale scores and low apathy composite scores would be placed correctly in the CBD group $88 \%$ of the time in this patient sample (sensitivity $67 \%$, specificity $97 \%$, overall accuracy $88 \%$ ).

The irritability of patients with CBD was significantly associated with disinhibition and apathy (table 2). Their apathy was associated with disinhibition and anxiety; their disinhibition was associated with aberrant motor behaviour and delusions; their delusions were

Table 3 Mean (SEM, \%) neuropsychiatric inventory (NPI) composite scores (maximum 12) of individual symptoms of patients with corticobasal degeneration and progressive supranuclear palsy, and healthy controls

\begin{tabular}{llll}
\hline $\begin{array}{l}\text { Behaviour (NPI composite } \\
\text { scores) }\end{array}$ & $\begin{array}{l}\text { Corticobasal } \\
\text { degeneration }(n=15)\end{array}$ & $\begin{array}{l}\text { Progressive } \\
\text { supranuclear palsy } \\
(n=34)\end{array}$ & $\begin{array}{l}\text { Healthy controls } \\
(n=25)\end{array}$ \\
\hline Depression & $3.4(0.9,73)^{\star} \dagger$ & $0.5(0.3,18)$ & $0.24(0.3,4)$ \\
Apathy & $1.8(0.9,40)$ & $6(0.7,82)^{\star} \dagger$ & 0 \\
Irritability & $1.13(0.7,20) \dagger$ & $0.15(0.09,9)$ & $0.08(0.08,4)$ \\
Agitation & $0.27(0.2,20)$ & $0.06(0.06,3)$ & 0 \\
Anxiety & $1.1(0.7,13)$ & $0.24(0.1,12)$ & 0 \\
Disinhibition & $1.1(0.7,13)$ & $1.8(0.6,35)$ & $0.16(0.2,4)$ \\
Delusions & $0.2(0.2,7)$ & 0 & 0 \\
Aberrant motor behaviour & $0.07(0.06,7)$ & $0.47(0.4,6)$ & 0 \\
Euphoria & 0 & 0 & 0 \\
Hallucinations & 0 & 0 & 0 \\
Total NPI score & $9(3.2,87)$ & $9.2(1.2,88)$ & $0.5(0.4,1) \ddagger$ \\
\hline
\end{tabular}

${ }^{\star} \mathrm{p}<0.05$, patients with progressive supranuclear palsy $v$ patients with corticobasal degeneration; $\mathrm{tp}<0.05$ either patient group $v$ controls; $\ddagger \mathrm{p}<0.05$, both patient groups $v$ controls. associated with aberrant motor behaviour; and their anxiety was associated with delusions and aberrant motor behaviour. Although motor disturbances were not associated with any behaviour, they were associated with total MMSE, suggesting that some patients with CBD with the poorest motor function (higher scores) had better cognitive performance (higher total MMSE scores). A factor analysis was performed to investigate whether the strength of some correlations (for example, disinhibition, anxiety, apathy, irritability, aberrant motor behaviour) may suggest that these behaviours belong to a single syndrome rather than being independent behavioural domains. The analysis showed that these behaviours could be grouped into two factors: (1) irritability, disinhibition, and apathy and (2) anxiety and aberrant motor behaviour $(\mathrm{p}<0.0001)$. When we also included agitation and depression in the analysis, these behaviours were grouped into two factors: (1) agitation, apathy, disinhibition, irritability, and aberrant motor behaviour and (2) depression $(p<0.0001)$. Anxiety was included with both but more with factor 1 .

Patients with CBD with left motor symptoms $(n=7)$ had higher disinhibition (2 (SEM 1)), apathy (2.8 (SEM 2)) and irritability (2 (SEM 1)), and lower depression scores (2.4 (SEM 1)) than those with right motor symptoms ( $\mathrm{n}=8$; disinhibition, 0; apathy, 0.7 (SEM 0.6); irritability, 0.14 (SEM 0.1); depression, 4.6 (SEM 1.4), but none of these differences were significant.

\section{Discussion}

This is the first study to systematically investigate the neuropsychiatric disturbances of patients with CBD. We found that most $(87 \%)$ patients with CBD manifested neuropsychiatric symptoms, mostly depression (73\%), apathy (40\%), irritability $(20 \%)$, and agitation $(20 \%)$, and less commonly, anxiety, disinhibition, aberrant motor behaviours, or delusions. No patients evidenced euphoria or hallucinations as reported by caregivers on the NPI. Patients with CBD showed significantly more depression than both patients with PSP and controls; conversely, patients with PSP were more apathetic. Behaviouraly, patients with CBD were more likely (higher odds ratios) than those with PSP 
to exhibit depression and irritability in the absence of, or with low levels of, apathy. In fact, these features correctly identified the patients with CBD $88 \%$ of the time. However, patients with CBD also exhibited apathy which was unrelated to their depression. Apathy was manifested by inattention to usual interests, activities, or plans of family members, and by being less spontaneous and active than usual. Apathy of patients with CBD was associated with irritability, disinhibition, and anxiety suggesting possible common pathogenetic mechanisms. Irritability of patients with $\mathrm{CBD}$ was manifested by flashes of anger, impatience, being argumentative and difficult to get along with, unrelated to the frustration of their illness, and different from the patient's usual behaviour. Disinhibition was characterised by acting impulsively without considering the consequences and by being tactless or making crude remarks. Although, it could be argued that the combination of these features is counterintuitive, a factor analysis suggested that irritability, disinhibition, and apathy may belong to a single syndrome rather than being independent behavioural domains. This factor contains items indicative of frontal lobe dysfunction. In support of our findings, a recent study confirmed by necropsy showed that $58 \%$ of the patients with CBD studied had developed apathy, irritability, or disinhibition, often associated with frontal lobe release signs. ${ }^{16}$

In the current study, depression was the most frequent neuropsychiatric symptom exhibited by patients with CBD. These results differ from those of our necropsy study in which only a few $(7 \%)$ patients with CBD presented with depression at onset, although at least one third developed it during progression of disease. ${ }^{16}$ Neuropsychiatric symptoms are usually not thoroughly ascertained, and necropsy studies probably underestimate their presence. We did not determine if our patients' depression was reactive to their deficits, but the fact that it may antedate motor symptomatology (and was the first symptom in one of the 15 patients) indicates that at least in some patients with CBD, depression has a neurobiological basis. Depression in CBD was unrelated to any other behaviour, suggesting that its pathophysiological mechanism differed. Depression often occurs in other basal ganglia disorders such as Parkinson's disease and Huntington's disease, in which it may predate other symptoms. ${ }^{27-32}$ Depressed patients with Parkinson's disease have more severe neuronal loss in the dorsal raphe than non-depressed patients ${ }^{33}$; and in an ${ }^{18}$ F-fluoro-2-deoxyglucose $\left({ }^{18} \mathrm{~F}\right.$-FDG) PET study, there was significant hypometabolism in the caudate and orbital inferior frontal lobes. ${ }^{34}$ In addition, the depression scores of the patients with Parkinson's disease inversely correlated with the decreased metabolic activity (lower metabolism correlated with greater depression). ${ }^{34}$ Similarly, in an ${ }^{18}$ F-FDG PET study, depressed patients with Huntington's disease showed orbitofrontal and thalamic hypometabolism. ${ }^{35}$ Studies with ${ }^{18}$ F-FDG PET in $\mathrm{CBD}$ disclose diminished metabolic activities in the frontal, temporal, sensorimotor, and parietal association cortices as well as in the caudate nucleus, lenticular nucleus, and thalamus. ${ }^{36}{ }^{37}$ Studies with PET of regional oxygen uptake show significantly depressed values in the superior and posterior temporal, inferior parietal, and occipital cortices. In addition, there were significant abnormalities in the superior frontal, midfrontal, inferior frontal, frontal polar, and orbitofrontal areas of the prefrontal cortex. ${ }^{38}$ Striatal fluorodopa uptake measured with PET discloses diminished fluorodopa uptake in both the caudate and putamen. These metabolic abnormalities tend to be asymmetric with greater involvement contralateral to the most affected side of the body. The regions affected are similar to those described as abnormal in patients with other neurologically based depression syndromes, although no specific metabolic studies of patients with CBD with and without depression have been reported. Pathological changes in CBD include involvement of several nuclei of frontosubcortical circuits posited to mediate cognitive and emotional functions. Affected areas include the prefrontal cortex, globus pallidus, subthalamic nucleus, substantia nigra, locus coeruleus, raphe nuclei, and midbrain tegmentum. ${ }^{39}$ Biochemical deficits in CBD include a marked loss of noradrenaline and serotonin as well as a severe nigrostriatal dopamine deficiency. ${ }^{40}$ Similar transmitter deficiencies characterise other basal ganglia disorders with depression such as Parkinson's disease. By contrast, in PSP the locus coeruleus and raphe nuclei are relatively preserved, and noradrenaline and serotonin concentrations are usually not affected. Additionally, in PSP, by contrast to what occurs in CBD and Parkinson's disease, involvement of the frontosubcortical circuits is bilateral and symmetric. Whether unilateral or asymmetric involvement of the orbitofrontal circuit is needed to manifest biological depression, and bilateral involvement of the medial frontal circuits is necessary for the presence of apathy, need further evaluation.

Dysfunction of subcortical projections to premotor-motor and supplementary motor subcortical circuits explains the motor abnormalities exhibited by patients with CBD. Although in our study, patients' symptom duration was associated with disinhibition $(r=0.53)$ and motor scores $(r=0.53)$, motor disturbances were unrelated to any behaviour or specific cognitive disturbance (for example, frontal dysfunction). Moreover, patients with CBD with worse motor dysfunction (higher scores) had less cognitive impairment (higher total MMSE scores), suggesting that the various frontosubcortical pathways in CBD are not affected in parallel, at relatively early stages of the disease. Because this finding was made in a cross sectional, not a longitudinal study, our results do not disagree with the previous studies, ${ }^{16}$ which reported that cognitive symptoms progressed during the course of the disease. In support of our findings, we found that in cases confirmed by necropsy ${ }^{16}$ (unpublished material), at early disease stages, motor dysfunction was inversely associated with cognitive impair- 
ment (unilateral rigidity was inversely correlated with impaired cognition during the first 3 years $(r=-0.57, \mathrm{p}<0.05)$; and unilateral dystonia was inversely related to cognitive disturbances at onset $(r=-0.63, \mathrm{p}<0.01))$. Moreover, in the same data set, ${ }^{16}$ bilateral motor disturbances were positively associated with cognitive disturbances (bilateral bradykinesia was associated with impaired cognition during the first 3 years $(r=0.58, \mathrm{p}<0.05))$. These results suggest that at onset, patients may manifest either motor or cognitive disturbances which reflect the presence of neuronal degeneration in either motor or cognitive circuits. However, when motor symptoms are bilateral, usually with progression of the disease, patients show more obvious cognitive impairment. Because we evaluated patients at relatively early stages of their disease and used the score of the worse limb for the motor assessment, we found that at disease onset, degeneration of the different frontosubcortical circuits does not occur in parallel. It is not surprising that at early stages of the disease, the restricted cognitive disturbances patients exhibited hardly affected their performance on tests which evaluate overall mental status such as the MMSE.

This study has several limitations. It was based on small sample size, thus limiting the generalisation of our findings. In addition, our psychiatric diagnoses were based mainly on a neuropsychiatric rating scale and not on clinical diagnoses. Future studies would benefit from including a larger sample and complete clinical and neuropsychiatric evaluations.

Neuropsychiatric assessment may be helpful to better understand CBD and to better manage patients with this disorder. Future studies need to investigate whether depression in patients with CBD may benefit from treatment with antidepressant agents.

We are grateful to the patients and their relatives for their kind cooperation in our research project and to Mrs Devee Schoenberg for her skillful editorial assistance. This study was supported by an NIA Alzheimer Disease Center grant (AG 10123) and the Sidell-Kogan Foundation. The work was presented in part at the 122nd Annual Meeting of the American Neurological Association, San Diego, California, 28 September to 1 October 1997.

1 Rinne JO, Lee MS, Thompson PD, et al. Corticobasal degeneration. A clinical study of 36 cases. Brain 1994;117:1183-96.

2 Litvan I, Agid Y, Goetz C, et al. Accuracy of the clinical diagnosis of corticobasal degeneration: a clinicopathologidiagnosis of corticobasal degeneration:
cal study. Neurology 1997;48:119-25.

3 Feany MB, Dickson DW. Widespread cytoskeletal pathology characterizes corticobasal degeneration. Am f Pathol ogy characterizes cort

4 Lang AE, Bergeron C, Pollanen MS, et al. Parietal Pick's disease mimicking cortical-basal ganglionic degeneration. Neurology 1994;44:1436-40.

5 Jendroska K, Rossor MN, Mathias CJ, et al. Morphological overlap between corticobasal degeneration and Pick's disease: a clinicopathological report. Mov Disord 1995;10: $111-4$.

6 Bergeron C, Pollanen MS, Weyer L, et al. Unusual clinical presentations of cortical-basal ganglionic degeneration. Ann Neurol 1996;40:893-900.

7 Feany MB, Mattiace LA, Dickson DW. Neuropathologic overlap of progressive supranuclear palsy, Pick's disease, and corticobasal degeneration. I Neuropathol Exp Neurol 1996;55:53-67.

8 Bergeron C, Pollanen MS, Weyer L, et al. Cortical degeneration in progressive supranuclear palsy. A comparidegeneration in progressive supranuclear palsy. A comparison with cortical-basal ganglionic degent
ropathol Exp Neurology 1997;56:726-34

9 Schneider JA, Watts RL, Gearing M, et al. Corticobasal degeneration: neuropathologic and clinical heterogeneity. Neurology 1997;48:959-69.
10 Brunt ER, van Weerden TW, Pruim J, et al. Unique myoclonic pattern in corticobasal degeneration. Mov myoclonic pattern in
Disord 1995;10:132-42.

11 Thompson PD, Day BL, Rothwell JC, et al. The myoclonus in corticobasal degeneration. Evidence for two forms of cortical reflex myoclonus. Brain 1994;117:1197-207.

12 Leiguarda R, Lees AJ, Merello $\mathrm{M}$, et al. The nature of apraxia in corticobasal degeneration. I Neurol Neurosurg Psychiatry 1994;57:455-9.

13 Pillon B, Blin J, Vidailhet $M$, et al. The neuropsychological pattern of corticobasal degeneration: comparison with progressive supranuclear palsy and Alzheimer's disease. Neurology 1995;45:1477-83.

14 Vidailhet M, Rivaud S, Gouider-Khouja N, et al. Eye movements in parkinsonian syndromes. Ann Neurol 1994;35: 420-6.

15 Rey GJ, Tomer R, Levin BE, et al. Psychiatric symptoms, atypical dementia, and left visual field inattention in corticobasal ganglionic degeneration. Mov Disord 1995;10:10610 .

16 Wenning GK, Litvan I, Jankovic J, et al. Natural history and survival of 14 corticobasal degeneration cases: a clinicopathologic study. F Neurol Neurosurg Psychiatry 1998, 64:184-9.

17 Steele C, Rovner B, Chase GA, et al. Psychiatric symptoms and nursing home placement of patients with Alzheimer's disease. Am f Psychiatry 1990;147:1049-51.

18 Morriss RK, Rovner BW, Folstein MF, et al. Delusions in newly admitted residents of nursing homes. Am f Psychiatry 1990;147:299-302.

19 Goetz CG, Stebbins GT. Risk factors for nursing home placement in advanced Parkinson's disease. Neurology 1993;43:2227-9.

20 Goetz CG, Stebbins GT. Mortality and hallucinations in nursing home patients with advanced Parkinson's disease. Neurology 1995;45:669-71.

21 Cummings JL, Mega M, Gray K, et al. The Neuropsychiatric Inventory: comprehensive assessment of psychopathology in dementia. Neurology 1994;44:2308-14.

22 Litvan I, Mega MS, Cummings JL, et al. Neuropsychiatric aspects of progressive supranuclear palsy. Neurology 1997; 47:1184-9.

23 Folstein MF, Folstein SE, McHugh PR. Mini-mental state: a practical method for grading the mental state of patients for the clinician. 7 Psychiatr Res 1975;12:189-98.

24 Mattis S. Mental status examination for organic mental syndrome in the elderly patient. In: Bellak L, Karasu TB, eds. Geriatric psychiatry. New York: Grune and Stratton, 1976.

25 Fahn S, Elton RL, and members of the UPDRS Committee. Unified Parkinson's disease rating scale. Florham Park, NJ: Macmillan Healthcare Information, 1987:153-64.

26 Efron B, Tibshirani R. Statistical data analysis in the computer age. Science 1991;253:390-5.

27 Santamaria J, Tolosa E, Valles A. Parkinson's disease with depression: a possible subgroup of idiopathic parkinsonism. Neurology 1986;36:1130-3.

28 Cummings JL. Depression and Parkinson's disease: a review. Am ₹ Psychiatry 1992;149:443-54.

29 Cummings JL, Diaz C, Levy M, et al. Neuropsychiatric syndromes in neurodegenerative diseases: frequency and significance. Seminars in Clinical Neuropsychiatry 1996;1: 241-7.

30 Starkstein SE, Preziosi TJ, Bolduc PL, et al. Depression in Parkinson's disease. 7 Nerv Ment Dis 1990;178:27-31.

31 Starkstein SE, Mayberg HS, Leiguarda R, et al. A prospective longitudinal study of depression, cognitive decline, and physical impairments in patients with Parkinson's disease. $\mathcal{F}$ Neurol Neurosurg Psychiatry 1992;55:377-82.

32 Tandberg E, Larsen JP, Aarsland D, et al. The occurrence of depression in Parkinson's disease. A community-based study. Arch Neurol 1996;53:175-9.

33 Paulus W, Jellinger K. The neuropathologic basis of different clinical subgroups of Parkinson's disease. $\mathcal{F}$ Neuropathol Exp Neurol 1991;50:743-55.

34 Mayberg HS, Starkstein SE, Sadzot B, et al. Selective hypometabolism in the inferior frontal lobe in depressed patients with Parkinson's disease. Ann Neurol 1990;28:57patien.

35 Mayberg HS, Starkstein SE, Peyser CE, et al. Paralimbic frontal lobe hypometabolism in depression associated with Huntington's disease. Neurology 1992;42:1791-7.

36 Blin J, Vidaihlet MJ, Pillon B, et al. Corticobasal degeneration: decreased and asymmetrical glucose consumption as studied with PET. Mov Disord 1992;7:348-54.

37 Nagashama Y, Fukuyama H, Turjanski N, et al. Cerebral glucose metabolism in corticobasal degeneration: comparison with progressive supranuclear palsy and normal controls. Mov Disord 1997;12:691-6.

38 Sawle GV, Brooks DJ, Marsden CD, et al. Corticobasal degeneration. A unique pattern of regional cortical oxygen hypometabolism and striatal fluorodopa uptake demonstrated by positron emission tomography. Brain 1991;114: 541-56.

39 Gibb WR, Luthert PJ, Marsden CD. Corticobasal degeneration. Brain 1989;112:1171-92.

40 Kish SJ, Gilbert JJ, Chang LJ, et al. Brain neurotransmitter abnormalities in neuronal intranuclear inclusion body disorder. Ann Neurol 1985;17:405-7. 